\title{
Molecular fine-tuning of affinity maturation in germinal centers
}

\author{
Yee Ling Wu and Cristina Rada \\ MRC Laboratory of Molecular Biology, Cambridge, United Kingdom.
}

The development of high-affinity antibodies in response to infection is an iterative process in which B cells cycle between proliferation/somatic hypermutation and antigen-driven selection. These processes occur within specific regions of the secondary lymphoid structures known as germinal centers (CCs) and the environmental and signaling cues provided by these regions guide the $\mathrm{CC}$ reactions that drive $B$ cell maturation and antibody production, ultimately determining B cell fate. In this issue of the $J C I$, Nakagawa and colleagues examine the role of miR-155, a microRNA that is required for CC development and the production of high-affinity antibodies. They show that miR-155 is highly expressed in positively selected B cells and promotes survival of these cells by orienting the Myc transcription program toward survival rather than apoptosis through the inhibition of the transcriptional regulator JARID2. These findings illustrate the fine balance between apoptosis and proliferation that is required for the development of high-affinity antibodies.
The germinal center response and affinity maturation

The development of high-affinity antibodies in response to pathogens is key to the acquisition of immune protection and to the establishment of long-term immunity. The development of high-affinity antibodies occurs in organized histologic structures within secondary lymphoid tissues, known as germinal centers (GCs). GCs emerge from clusters of proliferating $\mathrm{B}$ cells in response to pathogen-derived antigens with the cooperation of signals from $\mathrm{T}$ cells and antigen presenting cells (APCs). High-affinity antibodies develop by an iterative process of mutation and selection, where the genes encoding B cell antigen receptors (BCRs) are bombarded with point mutations and subsequently tested for their ability to bind antigen in competition with unmutated antigen receptors and, possibly, preexisting antibodies. In GCs, B cells are selected based on the affinity of the BCR for antigen ligands.
The speed and efficiency of the antibody response, particularly to rapidly dividing pathogens, rely on the exquisite regulation of cell fate during the temporal maturation of the GC response. This requires $\mathrm{B}$ cells to finely balance proliferation, differentiation, and cell death in response to external cues from the microenvironment. The spatial segregation of these cues is key to the process. Upon stimulation through antigen-specific BCRs and the costimulatory receptor CD40, B cells expand in the so-called dark zone (DZ) of the GC, where rapid proliferation is coupled with somatic hypermutation. DZ B cells stop mutating and dividing and then transit to the light zone (LZ), where competition for antigen from immune complexes anchored on the surface of follicular dendritic cells (FDCs) and interaction with $\mathrm{T}$ cells provide survival signals. In the absence of survival signals, B cells execute a default apoptotic program. Multiple

Related Article: p. 377

Conflict of interest: The authors have declared that no conflict of interest exists.

Reference information: / Clin Invest. 2016;126(1):32-34. doi:10.1172/JCI85627.

iterations of these cycles of proliferation/ hypermutation and antigen-driven selection result in $\mathrm{B}$ cells bearing BCRs with increased affinity; in turn, these selected B cells can differentiate into memory B cells or antibody-producing plasma cells.

\section{Modulating cell fate decisions in the $\mathrm{GC}$ reaction}

Recent investigations have clarified the architecture of the GC and the signals that drive the maturation of the $\mathrm{GC}$ reaction to enhance our understanding of how cellto-cell interactions, the chemokine environment, and antigen itself control GC B cell fates (1). However, how these extrinsic signals are integrated by individual GC B cells remains unclear, as do the molecular pathways that determine GC B cell fates: reentry into the $\mathrm{DZ}$, differentiation into memory or antibody-secreting plasma cells, or initiation of apoptosis.

In this issue, Nakagawa and colleagues (2) address an important question regarding the molecular basis for the regulation of affinity maturation during the GC reaction. The work centers on the role of miR155 , a microRNA expressed in hematopoietic cells, including B and T lymphocytes and myeloid cells (reviewed in ref. 3). miR155 is induced in activated B cells, and its absence results in poorly developed GCs and impaired production of high-affinity antibodies $(4,5)$. However, the role of miR155 is complex because it regulates multiple gene targets and acts in more than just B cells. To overcome this limitation, the authors employed a well-established methodology in which WT or miR-155deficient hen egg lysozyme (HEL) antigenspecific B cells were transferred into congenic mice. They then followed the fate of these cells in the different compartments in the context of ongoing HEL-specific GC responses. This allowed them not only to test the cell-autonomous effect of miR-155 deficiency, but also to follow the expression of a key regulator of cell proliferation - the proto-oncogene Myc - along with 
A

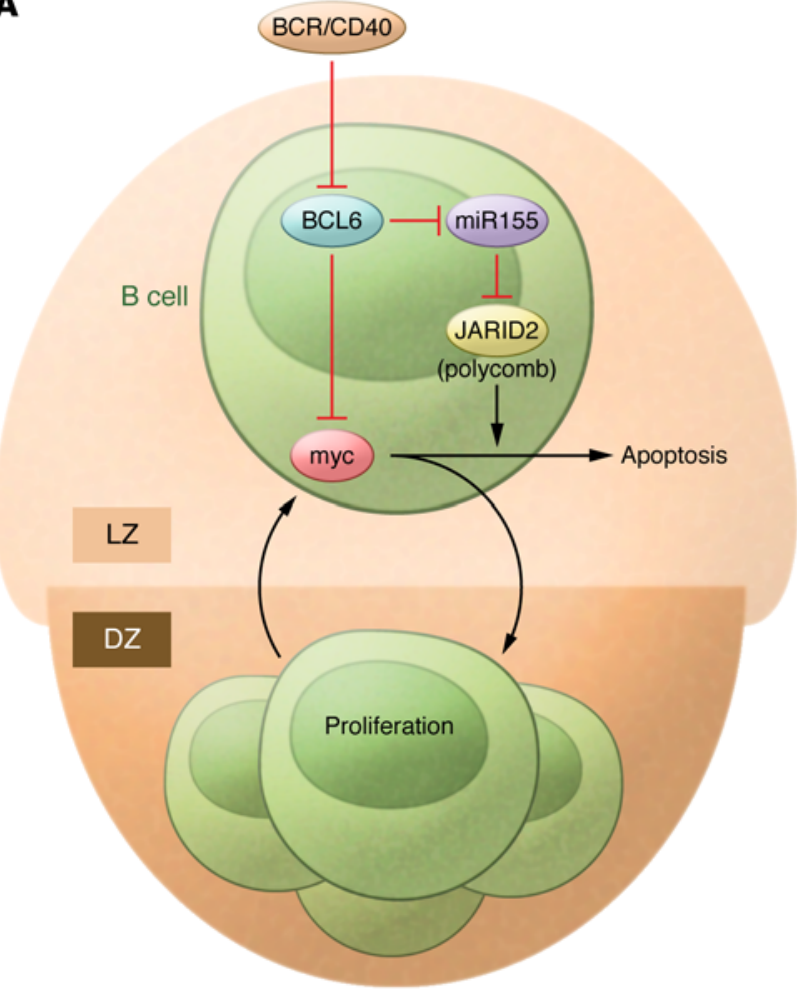

B

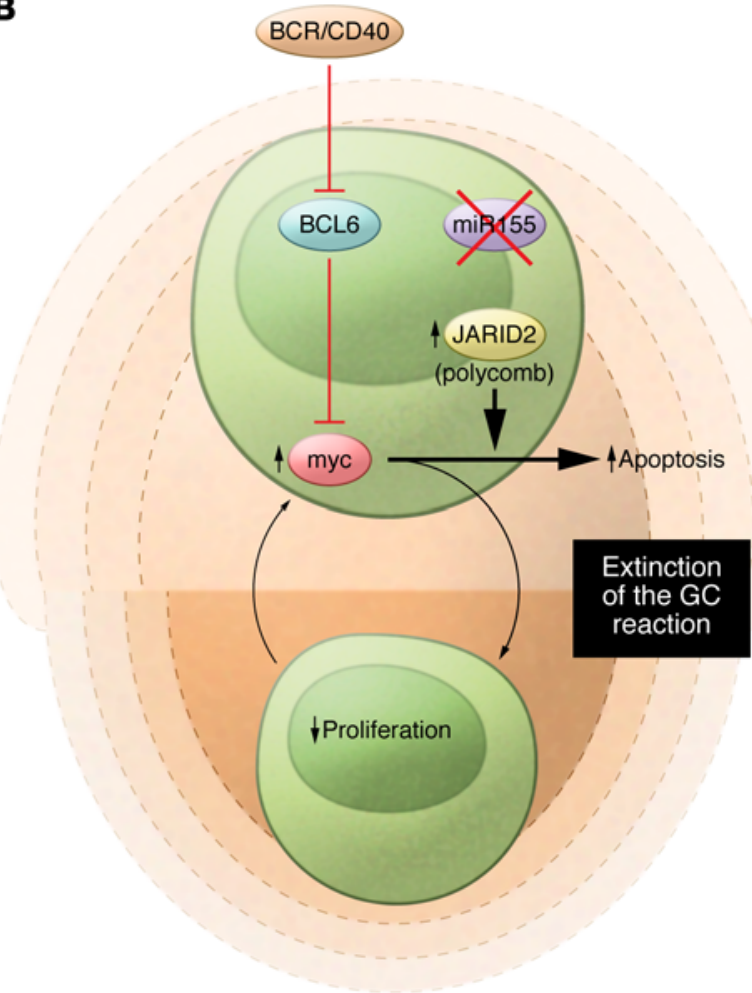

Figure 1. Molecular control of the Myc program in the GC LZ during antigen selection. (A) In mature GCs, a subset of LZ B cells expresses high levels of Myc. These cells downregulate BCL6 upon integration of signals from T cells and antigen through the BCR and CD40, respectively, allowing Myc and miR-155 to accumulate. Increased levels of Myc promote DZ reentry and division of the cells undergoing selection. Expression of the Myc transcriptional program also makes cells more sensitive to apoptosis, particularly in the absence of strong growth-promoting signals. Expression of miR-155 downregulates the levels of JARID2, which tips the Myc program toward proliferation rather than apoptosis. (B) In the absence of miR-155, selection is impaired, as increased apoptosis reduces the number of B cells capable of DZ reentry and eventually exhausts the GC reaction. The result is a failure to produce highaffinity antibodies in a timely fashion.

the expression of miR-155 itself at the single cell level by using reporters expressed from the respective endogenous loci.

It is well established that Myc plays a critical role in the biology of GC B cells, as it was one of the first translocation partners identified in B cell lymphomas of GC origin. Myc mediates its multiple roles by regulating a transcriptional network that is modulated by the differentiation status of the cell and is, therefore, able to promote proliferation, differentiation, or apoptosis depending on the context (6). Early in the initiation of the GC reaction, expression of a master transcriptional regulator, BCL6, suppresses Myc expression in GC B cells, except in a small number of B cells in the LZ. Nonetheless, low levels of Myc are essential to the GC reaction, since genetic ablation of Myc in GC cells results in the complete collapse of the GC reaction and failure of the affinity maturation of antibody responses (7). Indeed, Myc expression in this subset of
LZ B cells allows them to reenter the DZ and further proliferate, maintaining the cycles of proliferation and selection that are essential for the affinity-driven evolution of the $\mathrm{GC}$ reaction (8).

\section{Striking a balance with Myc and miR-155}

Nakagawa and colleagues identified a population of antigen-specific LZ B cells that express both Myc and high levels of miR155; this population exhibited increased cell cycle progression and apoptosis. More importantly, the levels of apoptosis were increased in the absence of miR-155 in the equivalent LZ B cell population. This increase in LZ apoptosis was also correlated with a deficit in the production of high-affinity, antigen-specific IgG1 GC B cells. The authors interpreted this finding as an indication that miR-155 counterbalances the induction of apoptosis mediated by Myc expression in the LZ B cells that are undergoing antigen selection.
Reduced apoptosis weakens the selective pressure on the LZ B cells, as exemplified by experimental overexpression of antiapoptotic genes like BCL2, which compromises the generation of high-affinity antibodies during GC responses (9). However, increased apoptosis, as in the case of miR-155-deficient B cells, also impairs affinity maturation. This observation was supported by mathematical modeling of the GC reaction, which suggested that a small increase in apoptosis could recapitulate the phenotype that they observed in vivo. Thus, high levels of Myc expression in LZ B cells undergoing antigen selection promotes proliferation (reentry into the DZ) and cell death, whereas high levels of miR-155 protects these cells from excessive apoptosis, preventing the collapse of the $\mathrm{GC}$ reaction.

These findings raise the question of how miR-155 orients the Myc transcriptional program toward survival rather than apoptosis. Nakagawa and colleagues 
identified a role for jumonji, AT rich interactive domain 2 (JARID2), a known target of miR-155 that has also been implicated in regulating gene expression (10). JARID2, as well as several other proteins crucial for affinity maturation in the GC - such as activation-induced cytidine deaminase (AICDA) or spleen focus forming virus proviral integration oncogene (SPI1) - have been independently validated as targets of miR-155 (11). Nakagawa and colleagues showed that overexpression of JARID2 affected the level of apoptosis of LZ antigen-specific cells to a similar degree as that observed for miR-155-deficient LZ B cells. Furthermore, they showed that JARID2 is normally downregulated in the subpopulation of LZ B cells that expresses high levels of miR-155, and that this downregulation is lost in miR-155-deficient cells. Finally, they showed that in miR155-deficient cells, downregulation of JARID2 did not have a measurable effect on the levels of apoptosis in LZ cells. Taken together, these results suggest that a functional interplay between JARID2 and miR-155 levels is required to determine the fate of the cell; they also suggest that suppressing JARID2 levels per se is only functionally relevant in the context of the normal differentiation program of positively selected LZ B cells and that miR-155 has additional influence on such programs independent of JARID2.

\section{Future directions: epigenetic alterations}

JARID2 is a cofactor of the polycomb repressive complex 2 (PRC2) chromatin modifier that mediates methylation of lysine 27 in histone H3 (H3K27me2/3), a modification that is associated with gene silencing. Although JARID2 has no enzymatic activity of its own, it promotes the recruitment of PRC2 to its target genes to induce gene silencing. However, the role of JARID2 is not completely clear. For example, JARID2-deficient ESCs do not derepress PRC2-silenced genes; instead, these cells suffer a severe block in their ability to differentiate (10). A similar phenotype was observed in JARID2-deficient fibroblasts and cardiomyocytes, which exhibited increased proliferation and impaired differentiation (12). The association of JARID2-dependent PRC2 target genes with chromatin configurations that are characteristic of bivalent promoter domains suggest that JARID2 expression levels might be important for cells confronted with a choice of transcriptional program, such as cells undertaking differentiation (12). This has been demonstrated in JARID2-deficient ESCs, where RNA polymerase II (RNAPII) recruitment to such bivalent promoters is impaired and transcription is compromised (13). Thus, it is apparent that JARID2 is required for the efficient initiation of transcription and not necessarily for the maintenance of gene silencing.

Polycomb genes have been implicated in the regulation of $\mathrm{B}$ cell maturation and differentiation in the GC and are differentially expressed in subcompartments of GC B cells. Moreover, dysregulation of polycomb genes, such as EZH2, is a common occurrence in GC-derived B cell lymphomas (reviewed in ref. 14). miR-155 has been shown to modulate the levels of JARID2 in the course of Th1 and Th17 responses to immune challenges, leading to changes in the expression of effector cytokines such as IL-10 or IL-17a (15). Thus, modest changes in the levels of JARID2 elicited by miR-155 can effect changes in the differentiation fate and transcriptional program of $\mathrm{T}$ cells.

The fine balance between promoting proliferation versus promoting the proapoptotic program in GC B cells undergoing selection induced by Myc appears to be modulated by JARID2 levels and potentially by the epigenetic landscape controlled by PRC2 (Figure 1). It would therefore be interesting to define the changes in PRC2 target genes that take place in LZ B cells undergoing selection and to determine whether these changes are also responsible for preventing differentiation toward plasma or memory cells. In the mean time, the work by Nakagawa and colleagues provides a critical advance in the mechanistic understanding of how affinity maturation is finely tuned during the GC reaction by balancing apoptosis and proliferation, and it implicates JARID2 and epigenetic regulation as one of the players in these processes.

\section{Acknowledgments}

Work in the authors' laboratory is supported by the Medical Research Council (MC_U105178806).

Address correspondence to: Cristina Rada, MRC Laboratory of Molecular Biology, Francis Crick Avenue, Cambridge CB2 OQH, United Kingdom. Phone: 44.0.1223.267098; E-mail: car@mrc-lmb.cam.ac.uk.

1. Victora GD, Nussenzweig MC. Germinal centers. Annu Rev Immunol. 2012;30:429-457.

2. Nakagawa R, et al. MicroRNA-155 controls affinitybased selection by protecting $\mathrm{c}-\mathrm{Myc}^{+} \mathrm{B}$ cells from apoptosis. JClin Invest. 2016;126(1):377-388.

3. Elton TS, Selemon H, Elton SM, Parinandi NL. Regulation of the MIR155 host gene in physiological and pathological processes. Gene. 2013;532(1):1-12.

4. Rodriguez A, et al. Requirement of bic/ microRNA-155 for normal immune function. Science. 2007;316(5824):608-611.

5. Vigorito E, et al. microRNA-155 regulates the generation of immunoglobulin class-switched plasma cells. Immunity. 2007;27(6):847-859.

6. McMahon SB. MYC and the control of apoptosis. Cold Spring Harb Perspect Med. 2014;4(7):a014407.

7. Calado DP, et al. The cell-cycle regulator c-Myc is essential for the formation and maintenance of germinal centers. Nat Immunol. 2012;13(11):1092-1100.

8. Dominguez-Sola $\mathrm{D}$, et al. The proto-oncogene MYC is required for selection in the germinal center and cyclic reentry. Nat Immunol. 2012;13(11):1083-1091.

9. Smith KG, Light A, O'Reilly LA, Ang SM, Strasser A, Tarlinton D. bcl-2 transgene expression inhibits apoptosis in the germinal center and reveals differences in the selection of memory B cells and bone marrow antibodyforming cells. JExp Med. 2000;191(3):475-484.

10. Li G, Margueron R, Ku M, Chambon P, Bernstein BE, Reinberg D. Jarid2 and PRC2, partners in regulating gene expression. Genes Dev. 2010;24(4):368-380.

11. Neilsen PM, et al. Mutant p53 drives invasion in breast tumors through up-regulation of miR-155. Oncogene. 2013;32(24):2992-3000.

12. Landeira D, Fisher AG. Inactive yet indispensable: the tale of Jarid2. Trends Cell Biol. 2011;21(2):74-80.

13. Landeira D, et al. Jarid2 is a PRC2 component in embryonic stem cells required for multi-lineage differentiation and recruitment of PRC1 and RNA Polymerase II to developmental regulators. Nat Cell Biol. 2010;12(6):618-624.

14. Wang GG, Konze KD, Tao J. Polycomb genes, miRNA, and their deregulation in B-cell malignancies. Blood. 2015;125(8):1217-1225.

15. Escobar TM, et al. miR-155 activates cytokine gene expression in Th17 cells by regulating the DNA-binding protein Jarid2 to relieve polycomb-mediated repression. Immunity. 2014;40(6):865-879. 